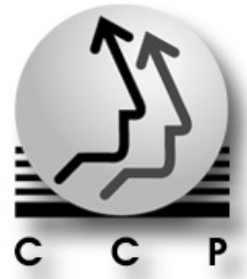

Población y Salud en Mesoamérica

Revista electrónica publicada por el

Centro Centroamericano de Población,

Universidad de Costa Rica, 2060 San José, Costa Rica

http://ccp.ucr.ac.cr

Población y Salud en Mesoamérica

Revista electrónica semestral, ISSN-1659-0201

Volumen 10, número 1 , artículo 2

Julio - diciembre, 2012

Publicado 1 de julio, 2012

http://ccp.ucr.ac.cr/revista/

\title{
Sobrepeso y obesidad en preescolares y escolares de una comunidad periurbana de origen otomí del Valle de Toluca, México
}

\author{
Carmen Liliana Ceballos Juárez \\ Ivonne Vizcarra Bordi \\ Liliana Diego Acosta \\ Carlos A. Reyes-Ortiz \\ Mariela Loza Torres
}

\section{(cc) EY-NG-ND}

Protegido bajo licencia Creative Commons

Centro Centroamericano de Población 


\title{
Sobrepeso y obesidad en preescolares y escolares de una comunidad periurbana de origen otomí del Valle de Toluca, México
}

\author{
Overweight and obesity in preschool and school children from \\ a periurban community of Otomi origin of the Toluca Valley, Mexico \\ Carmen Liliana Ceballos Juárez, Ivonne Vizcarra Bordi², Liliana Diego Acosta, Carlos A. \\ Reyes-Ortiz, Mariela Loza Torres ${ }^{3}$
}

\section{RESUMEN}

Este artículo parte de un objetivo que consistió en diagnosticar el estado nutricional y detectar la incidencia de sobrepeso y obesidad en preescolares y escolares de 4 a 16 años de edad de una comunidad periurbana de origen otomí del municipio de Toluca, Estado de México. Para determinar la prevalencia, se obtuvo el Índice de Masa Corporal (IMC) de 2208 preescolares y escolares de tres escuelas de diferentes niveles académicos, primaria y secundaria. En los tres niveles se analizaron las variables de sexo/género y edad, y solo en el nivel de secundaria se introdujo la variable étnica. Los resultados muestran que existen grados escolares de alerta (final de cada nivel escolar), donde la prevalencia de sobre peso aumenta, significativamente, para hombres escolares hasta la edad de 12 años. A nivel de secundaria, las mujeres son las que presentan mayor incidencia de sobrepeso y obesidad, pero el riesgo es mayor cuando no se tienen orígenes otomíes. Se concluye que el IMC puede ser un indicador útil para establecer medidas de prevención antes de los 16 años de edad, pero no suficiente. Es necesario incluir la circunferencia de cintura en la vigilancia nutricional desde la edad preescolar para tamizar a aquellos individuos con obesidad central como marcador de riesgo de enfermedad cardiovascular.

Palabras clave: sobrepeso, obesidad, población preescolar, población escolar, población indígena

\begin{abstract}
The aim of this article is to diagnose the nutritional status and detect insidencia of overweight and obesity in infants 4 to 16 years of age in an Otomi peri-urban town of Toluca, Mexico State. To determine the prevalence was obtained body mass index (BMI) of 2208 preschoolers and schoolchildren in three schools from different academic levels, preschool, primary and secondary school. At all three levels were analyzed on sex / gender and age, and only in the secondary level was introduced ethnicity. The results show that existing warning grades (end of each grade level) where the prevalence of overweight increased significantly for men school until the age of 12. At the secondary level are women who have higher incidence of overweight and obesity, but the risk is greater when there is no Otomi origin. We conclude that BMI can be a useful indicator for preventive strategies before 16 years of age, but not sufficient, it is necessary to include waist circumference in nutritional surveillance from preschool to screen those individuals with central obesity as a risk marker for cardiovascular disease
\end{abstract}

Keywords: overweight, obesity, preschool population, school population, indigeneus population.

Recibido: 18 dic. 2011

Aprobado: 29 mar. 2012

\footnotetext{
${ }^{1}$ Universidad Autónoma del Estado de México, Facultad de Medicina. MEXICO. lili_2811@hotmail.com

${ }^{2}$ Universidad Autónoma del Estado de México, Instituto de Ciencias Agropecuarias y Rurales. MEXICO. ivbordi@yahoo.com.mx

${ }^{3}$ Universidad Autónoma del Estado de México, Instituto de Ciencias Agropecuarias y Rurales. MEXICO m_1_nais@yahoo.com
} 


\section{INTRODUCCIÓN}

Durante los últimos años, en conjunto con el proceso de globalización, han ocurrido importantes transformaciones demográficas, socioculturales, tecnológicas y económicas a nivel mundial. Sus consecuencias más visibles han sido las modificaciones en el perfil epidemiológico, en los estilos de vida, en los patrones de consumo alimentario y en las tasas de morbi-mortalidad de las sociedades latinoamericanas (Peña y Bacallao, 2000; Angarita et al., 2001, Vizcarra y Lutz, 2010), entre las que destacan una disminución de las patologías de origen infeccioso y un progresivo incremento de las enfermedades crónico-degenerativas no transmisibles, como la obesidad (Peña y Bacallao, 2000). Por su magnitud y rápido crecimiento de su prevalencia en poblaciones cada vez más jóvenes, la obesidad ha comenzado a tratarse como una epidemia mundial (Violante Ortiz, 2001), repercutiendo directamente en la población infantil de todos los niveles socioeconómicos, incluidos aquellos sectores que no han superado la pobreza, sino que han sostenido o han deteriorado su nivel socioeconómico (Solano et al., 2005).

Paradójicamente, en muchos de estos bajos sectores, como las poblaciones indígenas, prevalecen altas tasas de desnutrición infantil registradas desde hace décadas (Psacharopoulos y Patrinos., 1994; Gotthelf et al., 2004). Para el caso de México, se ha observado que en poblaciones pobres rurales e indígenas beneficiarias de programas asistenciales para mejorar la alimentación y la nutrición infantil, coexisten la desnutrición infantil y el sobrepeso u obesidad en la edad adulta (CONEVAL, 2009; Conzuelo y Vizcarra, 2009).

No obstante, la realidad sugiere que el problema actual de salud infantil es el sobrepeso y la obesidad, convirtiéndose estas patologías en un problema social y de salud pública (Carmona y Vizcarra, 2009). Por un lado, porque dan paso a otras patologías de carácter crónico como diabetes mellitus, hipertensión arterial, dislipidemias, entre otras, que si bien antes se presentaban en edades adultas, hoy en día niños y adolescentes las padecen. Por otro lado, porque el estado nutricional de niños(as) y adolescentes que se encuentran en edades donde se produce el máximo desarrollo corporal, también adquieren los comportamientos sociales que guiarán sus estilos y hábitos de vida y, por lo tanto, su perfil en el estado de salud en la edad productiva o laboral (Solano et al., 2005).

En poblaciones indígenas que han vivido transformaciones como la urbanización, la obesidad va adquiriendo particularidades poco estudiadas en México. Se sabe que en poblaciones de aborígenes o autóctonas urbanas en América del Norte (Canadá y los Estados Unidos de Norteamérica), la prevalencia de obesidad infantil y de adolescentes se ha duplicado en la última década, adquiriendo una velocidad más rápida que en el resto de los países donde radican, no solo por los hábitos adquiridos provenientes de los estilos de vida occidentales, sino por su condición de vulnerabilidad étnica y ambiental en la que se encuentran (Bernard et al., 2000; Caballero et al., 2003; Halpern, 2007). Sin embargo, en el estudio reciente de Bustos et al., (2009) en niños chilenos, la pobreza resultó ser un factor de riesgo de estatura baja, pero protector de obesidad independiente de la etnia, lo cual no descarta que la baja prevalencia de obesidad en escolares indígenas deba marginarse, pues una década atrás la obesidad no estaba presente en esas poblaciones.

Por lo anterior, es de nuestro interés dar a conocer el estado nutricional y la incidencia de 
sobrepeso y obesidad encontrada de preescolares y escolares (preescolar, primaria y secundaria) de la comunidad de origen indígena Otomí de la localidad de San Andrés Cuexcontitlán, perteneciente al municipio de Toluca, Estado de México, México, por sus características de periurbanidad y procesos de globalización.

Para ello, se consideraron las siguientes premisas: a) la obesidad infantil se detecta a partir de que se sobrepasa el $20 \%$ del peso ideal y que la probabilidad de ser un(a) adulto(a) obeso(a) cuando se ha sido un(a) niño(a) obeso(a) es diferente según la edad de comienzo de dicha obesidad (Cole, 2004; Nestle, 2007); b) la obesidad que se inicia en la infancia puede tener peores consecuencias que la obesidad que se inicia en la edad adulta (Aseguinolaza et al., 2001; Dietz, 1997); c) se sabe que del $40 \%$ de los adultos con obesidad comenzaron a presentarla cuando se presentó el sobrepeso a partir de los 6 meses hasta los 7 años, y del 70\% para los que comenzaron con obesidad entre los 10 y los 13 años (Bastos et al., 2005); d). Además de la edad, se consideran la pertenencia a una etnia, la clase y el género, pues se sabe que deben ser ejes transversales en los estudios epidemiológicos (Krebs et al., 2007).

Reforzando el planteamiento, algunos estudios pediátricos han señalado que entre los 4 y 7 años es cuando los y las niños(as) bajan la velocidad en la que ganan peso, formando en su curva de crecimiento una ligera V, llamada "rebote de adiposidad" (Rolland-Cachera et al., 1984), esta disminución temprana en el IMC refleja una disminución correspondiente en la grasa subcutánea y el porcentaje de la edad de grasa corporal (Malina et al., 2004), pero a partir de los 7 años hasta los 13 adquieren mayor número de células adiposas, es decir, que la diferencia se explica porque las células que almacenan grasa se multiplican sobre todo en esta etapa de la vida (Bastos et al., 2005).

Cabe reconocer que existe una preocupación política en materia de salud y desarrollo social, por conocer cuándo se deben invertir más recursos públicos para prevenir la obesidad y otras enfermedades crónicas degenerativas relacionadas con la pobreza, con la condición étnica, con los malos hábitos alimenticios, con la falta de activación física y con la adquisición de estilos de vida urbanizados y occidentales. Este estudio pretende, asimismo, señalar la importancia que tiene conocer la prevalencia del estado nutricional a través del Índice de Masa Corporal (IMC), al menos una vez al año, como una sencilla herramienta para diagnosticar el desarrollo de la obesidad en la infancia y en la adolescencia (Pietrobelli et al., 1998), ya que el IMC tiene la ventaja de contabilizar tanto la altura como el peso del individuo. En la práctica, se ha demostrado que detectar si un menor está ganando demasiado peso y a una velocidad más rápida de lo normal para su altura, ayuda a que existan políticas de intervención eficaces para evitar que esa obesidad infantil y en adolescentes se convierta en un factor de riesgo para la misma obesidad mórbida en la edad adulta y otras enfermedades que se relacionan con el síndrome metabólico (Mei et al. 2002; Krebs et al., 2007). 


\section{MATERIALES Y MÉTODOS}

Se trata de un estudio transversal, descriptivo y cuantitativo. De enero a julio de 2010, se realizó el estudio con preescolares y escolares de la comunidad de San Andrés Cuexcontitlán, situada en el Municipio de Toluca, Estado de México. En 2005, de los 2969 hogares en la comunidad, 1870 hogares (63\%) fueron considerados de origen indígena, porque al menos una persona (casi siempre adulta), aún habla la lengua Otomí dentro del hogar y porque conservan algunas costumbres de esa etnia (CONAPO, 2006). En 2010 el número de viviendas subió a 3802 (CONAPO-INEGI, 2011), lo que no significa necesariamente que el número de hogares con al menos un integrante que hable la lengua otomí haya subido en la misma proporción. Por la expansión urbana y residencial hacia estas zonas, la población no indígena que habita en la comunidad ha aumentado. En 2005 la población era de 14,687 (48.9\% hombres, 51.1\% mujeres), de los cuales $28.66 \%$ aún conservaban la lengua (CONAPO, 2006), en el 2010 la población llegó a 18,005 (49.3\% hombres, $50.7 \%$ mujeres), cuya población hablante de lengua otomí fue de $22.2 \%$. (SEDESOL, 2012).

No obstante, las escuelas que se seleccionaron para el estudio se sitúan en el corazón de la comunidad y fueron construidas y habilitadas por los habitantes oriundos. Ciertamente, la mayoría de los y las estudiantes que ahí asisten dicen no hablar la lengua otomí, por ello, se decidió preguntarles si en su familia ascendiente (padre, madre, abuelos paternos y maternos) alguien aún hablaba el Otomí. Por desgracia, las únicas autoridades educativas que nos permitieron realizar es pregunta fue la escuela secundaria. Por eso, el factor de descendencia étnica solo pudo ser observado en una escuela.

Las escuelas estudiadas fueron:

- Jardín de Niños “Lic. Benito Juárez” con una matrícula total de 349 preescolares en el turno matutino.

- Escuela Primaria "Miguel Hidalgo y Costilla" con una matrícula total de 1268 escolares en el turno matutino.

- Escuela Secundaria Técnica Industrial y Comercial No. 69 "Gral. Vicente Guerrero" (ESTIC) con una matrícula total de 714 escolares en el turno matutino.

En primera instancia, se solicitó la autorización de los delegados de la comunidad y de las autoridades de cada una de las escuelas a través de oficios, así como de los padres o las madres de familia, mediante el consentimiento informado, para realizar el estudio con sus hijos e hijas de estas escuelas. La población total de las tres escuelas fue de 2331 quedando una población estudiada de 2208, siendo excluidos(as) 123 por ausentismo, debido, principalmente, a enfermedad. En el Cuadro N. ${ }^{\circ} 1$ se observa la población estudiada por escuela y sexo/género ${ }^{4}$.

\footnotetext{
${ }^{4}$ Consideramos colocar esta categoría para distinguir hombres y mujeres, cuya diferencia sexual asume la adscripción al género que se le asigna culturalmente a la persona al nacer (Lamas, 2002). Este sistema identifica todas la combinaciones que se han dado en las preferencias y diversidades sexuales; sin embargo, para este estudio solo incluimos dos sistemas: mujer-femenino y hombre-masculino, sin la intención de discriminar a la población que se asuma a otro sistema, pero por ser menores de edad, presumimos generalidades de la sociedad Otomí.
} 
A los y las preescolares y escolares se les realizaron mediciones antropométricas tales como peso y estatura. En particular, a los escolares de la escuela secundaria se tomó, además, la medida de la circunferencia de cintura, a parte, de la pregunta referente a su ascendencia de origen Otomí.

Las mediciones fueron realizadas por 4 personas previamente entrenadas. Para la toma del peso se les solicitó que fuera con el mínimo de ropa posible y sin zapatos, utilizando una báscula clínica Tanita con capacidad de $140 \mathrm{~kg}$, y con una precisión de $100 \mathrm{gr}$. La estatura se obtuvo con un estadímetro marca Seca. Para clasificar a preescolares y escolares de acuerdo con su Índice de Masa Corporal (IMC), cuya fórmula es Peso $\mathrm{Kg} /$ Estatura $\mathrm{cm}^{2}$, se consideraron los percentiles (p) emitidos por la NCHS, que indican: menos de p5: bajo peso, más de p85 y menos de p95: sobrepeso y más de p95: obesidad.

En el caso de los escolares de la secundaria, para la medición de la circunferencia de cintura se colocó la cinta métrica de fibra de vidrio alrededor del abdomen en el punto superior de la cresta iliaca, asegurándose que la cinta no apretara y que estuviera en paralelo con el piso. La medición se hizo al final de la expiración normal (NIH, 2009).

Todos los análisis estadísticos se realizaron utilizando el sistema SAS para Windows ${ }^{\circledR}$, versión 9.2 (SAS Institute, Inc., Cary, NC), y el nivel de significancia se fijó en $p<0,05$. Para describir las características de la población de estudio se utilizó estadística descriptiva (\% o media \pm desviación estándar). Para determinar las asociaciones bivariadas entre las características de la población de estudio y las categorías de clasificación de trabajo, se utilizó la prueba de Chicuadrado/Wald para las diferencias en las proporciones o la prueba de t para las diferencias en los promedios. Para probar la asociación entre las variables dependientes y variables independientes se utilizó la regresión lineal multivariada y análisis de regresión logística, cuando la variable predictor ( $\mathrm{Pr})$ es mayor que $\mathrm{Ch}^{2}$.

\section{RESULTADOS}

Se creó una gran base de datos para calcular el IMC por escuela, grado, sexo/género y edad de los preescolares y escolares estudiados. De un total de 2208 estudiantes de las tres escuelas que participaron en el estudio, 1124 (50.9\%) fueron mujeres y 1084 (49.09\%) fueron hombres (Cuadro N. ${ }^{\circ} 1$ ).

La edad promedio de los y las estudiantes por escuela fue de:

- Para preescolares de 5.06 años para ambos sexo/géneros. Con un rango de 4 a 6 años de edad en los dos grados $\left(2^{\circ}\right.$ y $\left.3^{\circ}\right)$.

- Para escolares a nivel de primaria de 8.38 años para las niñas y 8.61 años para los niños. Con un rango de 6 a 12 años de edad en seis grados.

- Para escolares a nivel de secundaría de 13.43 años para mujeres y 13.57 años para hombres. Con un rango de 12 a 15 años de edad en tres grados.

El Cuadro N. ${ }^{\circ} 2$ muestra las desviaciones estándar para cada escuela según el sexo/género, así como el concentrado de datos antropométricos. Se resalta que para cada grupo de datos (escuela y 
sexo/género F,M) el peso es el que mayor rango de variaciones tiene, y entre mayor es la edad este aumenta. En tal caso, los hombres (M) presentan mayor variación que sus pares femeninos (F) para cada una de las escuelas. Asimismo, se subraya que la desviación estándar de la circunferencia de la cintura de los hombres (M) de la escuela secundaria fue hasta más de dos veces superior que todos los demás datos, lo que sugiere que el sobrepeso y la obesidad no es unidimensional en cuanto a factores de edad y sexo/género, sino que, tal vez, refiere a multifactores complejos que tienen que ver tanto con los de orden clínico como los pscicososiales, económicos y ambientales.

\subsection{Preescolares del Jardín de Niños}

En el Cuadro N. ${ }^{3} 3$ se observan los resultados del IMC en promedio de preescolares, según el diagnóstico nutricional encontrado, afirmándose que el peso es el indicador que presenta mayor variabilidad. Por su parte, en el Cuadro N. ${ }^{\circ} 4$ se muestra la incidencia de cada uno de los cuatro diagnósticos nutricionales por sexo/género $(\mathrm{F}, \mathrm{M})$. Para bajo peso (p5), solo el 1.64\% de preescolares manifestó este nivel. En este caso, los niños presentan mayor dificultad para tener peso normal que sus pares niñas.

Si bien la mayoría tiene peso normal (p50), es decir, el $73.77 \%$ de la población estudiada para ambos sexo/género en promedio, se observa que la frecuencia de tener sobrepeso (p85) es más alta para las niñas que para los niños, pues el 20.13\% del total de las mujeres presentó este diagnóstico, en comparación con el $15.23 \%$ de los hombres. No obstante, la obesidad (p95) es más frecuente en los niños $(9.93 \%$ del total de la población masculina) que en las niñas $(3.9 \%$ del total de la población femenina).

De acuerdo con el coeficiente estimado (regresión logística) para la variable sexo/género, este es negativo y estadísticamente significativo ( $\angle 0,5)$, por lo que resultó ser una variable predictora de obesidad, para preescolares; se sugiere que las mujeres tendrían una menor probabilidad de presentar obesidad que sus pares masculinos (Cuadro N. ${ }^{\circ}$ ).

\subsection{Escolares de la Escuela Primaria}

Al igual que en los y las preescolares, mostramos en el Cuadro N. ${ }^{\circ} 6$ el diagnóstico nutricional de escolares, resaltando una vez más que la variable peso en cada estado nutricional es la que mayor tuvo desviación estándar, siendo la obesidad la de mayor importancia. Considerando la frecuencia por sexo/género, la prevalencia de tener bajo peso (p5) entre escolares es igualmente marginal (1.52\%) que en los preescolares; sin embargo, a comparación de los menores de 6 años, en la escuela primaria, las niñas presentan ligeramente más incidencia que los niños. Ahora bien, el peso normal (p50) sigue siendo el diagnóstico nutricional predominante entre los y las menores de 12 años de edad. Se encuentra que a nivel de primaria su prevalencia (68.48\%) es menor que en el Jardín de Niños, remarcando que los hombres tienen menor prevalencia en este estado nutricional y, por el contrario, ellos tienen la mayor prevalencia en sobrepeso (p85) y obesidad (p95) que las niñas, tal y como se muestra en el Cuadro N. ${ }^{\circ}$.

Lo anterior se confirma a través del estudio del modelo de regresión que se presenta en el cuadro 8. Ahí, el coeficiente estimado para la variable sexo/género, aparece negativo y es 
estadísticamente significativo ( $\angle 0,5)$, por lo que resultó ser nuevamente, una variable predictora de la obesidad. Al igual que en preescolares, con esta prueba se plantea que las mujeres tendrían una menor probabilidad de presentar obesidad que sus pares masculinos entre estudiantes de la escuela primaria.

\subsection{Escolares de la Escuela Secundaria}

Con la misma estructura de presentación de resultados hasta ahora, el Cuadro N. ${ }^{\circ} 9$ exhibe los diagnósticos nutricionales y las variables que las componen. Solo que a diferencia de las dos escuelas anteriores, es en este nivel escolar donde las desviaciones estándares de la variable cintura (circunferencia de cintura) se disparan hacia arriba con respecto a las otras variables, inclusive la de peso, la más notable en estudiantes de ambos sexo/género con un estado nutricional normal (p50).

Aún entre los 12 y 15 años de edad, el bajo peso (p5) sigue apareciendo con poca incidencia $(1.07 \%)$ dentro del cual, las mujeres tienen menor prevalencia que los hombres. De igual forma sucede en el estado nutricional normal, donde las mujeres presentan mayor incidencia para tener sobrepeso (p85 29.24\%), no así de obesidad (p95 9.94\%) con respecto a los hombres (11.46\%) (Cuadro N. $\left.{ }^{\circ} 10\right)$.

En el Cuadro N. ${ }^{\circ} 11$ se observan los resultados de las asociaciones bivariadas de sexo/género y/o edad a través de la prueba de Chi-cuadrado/Wald. En este se demuestra que en ninguna de las dos variables se obtuvo un coeficiente negativo y no fueron estadísticamente significativos $(\mathrm{p} \angle 0,5)$. En otras palabras, se sugiere que ni el sexo/género ni la edad intervienen en la predicción de la obesidad. Tal parece que llegada a la edad de la adolescencia, ambas variables son indistintas para presentar obesidad en esa edad (12 a 16 años).

\subsection{Escolares de la Escuela Secundaria con y sin origen otomí (indígena)}

Si bien es cierto que en nuestra muestra solo el $4.42 \%$ de los individuos que participaron en el estudio a nivel secundaria no tiene ningún arraigo a la cultura Otomí, aquí se quiso incluir la variable de diferenciación étnica, debido a que es un hecho el fenómeno del crecimiento acelerado de la urbanización en esta zona, por lo que es inevitable observar que habrán cada vez más escolares de otro origen conviviendo en los mismos espacios educativos que ofrece la comunidad y quienes aportarán y adoptarán nuevas conductas según sus propios estilos de vida. En este sentido exploratorio de diferenciación, se muestran en el Cuadro N. ${ }^{\circ} 12$ los datos antropométricos promedios según el origen (otomí o no). Ahí mismo, sobresale que el peso tiene mayor relevancia en las personas que no son oriundas y la circunferencia de la cintura varía más entre las personas con orígenes otomíes.

A través de un modelo de regresión lineal, se buscó si tener origen otomí, el sexo/género y/o la edad predecían el IMC. Se encontró que las tres variables fueron estadísticamente significativas ( $\mathrm{p} \angle 0,5)$, lo cual sugiere que la intersección de las tres variables predicen el IMC. Es decir, que tener origen Otomí implica tener un menor IMC que el no tener origen Otomí; a mayor edad hay más IMC; y las mujeres tienden a tener más IMC que los hombres (Cuadro N. ${ }^{\circ} 13$ ). 
Ahora bien, a través del modelo de regresión logística se buscó si tener origen otomí, según el sexo/género y la edad se podría predecir a la obesidad. Los hallazgos fueron que tener origen otomí sí predice a la obesidad por resultar estadísticamente significativo (p $\angle 0,5)$, indicando que tener orígenes otomíes puede tener menos probabilidad de obesidad que los que no provienen de la cultura otomíe; sin embargo, las otras dos variables (sexo/género y edad) no infieren en la predicción (Cuadro N. ${ }^{\circ} 14$ ).

\subsection{Prevalencias de obesidad y grados de alerta}

En resumen, y de acuerdo con el IMC, solo el 19.73\% del total de la población estudiada mostró tener sobrepeso (17.7\% en preescolar, $18.79 \%$ en primaria y $22.7 \%$ en secundaria); y $9.6 \%$ obesidad $(6.9 \%$ en preescolar, $11.22 \%$ en primaria y $10.7 \%$ en secundaria). Es importante destacar que en todos los grados preescolares y escolares estudiados, la prevalencia de sobrepeso es mayor que la de obesidad. No obstante, las prevalencias de obesidad que llaman la atención, por ser significativamente elevadas, se detectan en los grados escolares avanzados en cada escuela; $3^{\circ}$ del Jardín de Niños, de $3^{\circ}$ a $6^{\circ}$ de la Primaria, y $2^{\circ}$ y $3^{\circ}$ de la Secundaria (Cuadro N. $\left.{ }^{0} 15\right)$.

Por su parte, la Gráfica N. ${ }^{\circ} 1$ muestra que existe una pequeña curva de $\mathrm{V}$ entre la edad de 5 y 7 años al presentar sus promedios de IMC, según la media de edad por grado escolar. Los y las preescolares de tercer grado con edad promedio de 5.48 años, presentaron un IMC ligeramente mayor (18.37) que los escolares de primer grado de primaria con una edad promedio de 6 años (IMC 17.99). Prácticamente, a partir de esa edad existe una relación lineal ascendente entre IMC y edad promedios para todos los grados escolares estudiados.

Finalmente, vale la pena mencionar que mientras se tomaron las medidas antropométricas en las tres escuelas, se observó que en el caso del Jardín de Niños, la escuela sugiere, a través de un menú diseñado por una Licenciada en Nutrición a los padres de familia, el refrigerio que pudieran llevar sus hijos a la escuela, encontrando que la mayoría lo lleva a cabo, algunos se inclinan más por enviar productos industrializados altos en azúcares refinados y grasas saturadas. En el caso de la primaria y la secundaria, es más común la compra de alimentos en la cooperativa escolar que llevar refrigerio; también, se pudo constar que si bien hay disponibilidad de frutas y verduras, así como de alimentos preparados, su costo es mayor al de los productos tales como frituras, dulces, golosinas, refrescos, por mencionar algunos. 


\section{DISCUSIÓN}

Datos reportados por el Instituto Nacional de Salud Pública (INSP), en 2010, hacen evidente que los problemas de la nutrición en México, relacionados con la mala nutrición tanto por defecto como por exceso, constituyen problemas prioritarios de salud pública que afectan al individuo durante todo el ciclo de la vida y que tienen un alto costo sobre el capital humano y sobre la sociedad en su conjunto. Los resultados obtenidos en la presente investigación concuerdan con ello.

Ciertamente, estudios recientes en el medio rural del Estado de México han demostrado que la desnutrición infantil calórica-proteica y las enfermedades de origen metabólico y crónico degenerativas, no solo corresponden a una mala elección en los alimentos, sino también a factores estructurales como la transformación demográfica, la pérdida de tierras de cultivo para autoconsumo, el abandono del campo, la dependencia a un ingreso para la alimentación familiar, el desempleo, la migración, etc., (Vizcarra y Lutz, 2010). De aquí que el sobrepeso y la obesidad han adquirido gran importancia, debido a su rápido crecimiento en países de ingresos medios y bajos, padecimientos que, de no ser atendidos oportunamente, traen como consecuencia el incremento de la prevalencia de enfermedades crónico degenerativas (Cole, 2004; Nestle, 2007; Ortiz-Hernández y Cruz-Ángeles 2005).

En el presente estudio, el total de preescolares con sobrepeso y obesidad fue de $24.6 \%$, donde las niñas presentaron más sobrepeso y los niños más obesidad. En tanto que el total de escolares a nivel de la primaria con sobrepeso y obesidad fue de $30.01 \%$, de los cuales los hombres manifiestaron mayores prevalencias que sus pares femeninos. Tomando en cuenta que la obesidad comienza a edades tempranas y puede persistir hasta la edad adulta, como lo menciona Padrón (2002), resulta necesario no menoscabar cualquier incidencia de sobrepeso para intervenir adecuadamente en sistemas de salud preventivo según el sexo/género.

En México, los datos reportados por la Encuesta Nacional de Nutrición (ENN) de 1999 y del 2006 refieren que la prevalencia de sobrepeso y obesidad en escolares no se modificó, manteniéndose en $5.1 \%$ en ambos años, la diferencia radica en la prevalencia de sobrepeso y obesidad en la edad escolar, en el cual se marca una diferencia importante, apreciándose un aumento del 18.8\% al 26\%, es decir, que es un aumento del 7.2\% (Olaiz-Fernández et al. 2006). Los resultados de esta investigación concuerdan con el aumento de sobrepeso y obesidad que apareció en los escolares varones de primaria, siendo de $18.03 \%$ del total de la población estudiada de ambos sexos género de ese nivel educativo. Con mayor especificidad se asocia significativamente (0.0074) con el grado escolar, es decir, los niños que cursan el tercer y sexto grado presentan los porcentajes más altos de obesidad, por lo tanto, se aconseja que estos grados pueden ser tomados como "grados de alerta" para evitar la futura presencia de enfermedades crónico degenerativas asociadas a la obesidad.

Además, en nuestro estudio encontramos que entre los 4 y 7 años es cuando los y las preescolares y escolares tienen una disminución en el IMC (de 5 a 6 años de edad, formando una ligera V, llamada "rebote de adiposidad") (Rolland-Cachera et al., 1984), lo que sugiere otro foco de alarma para evitar la adiposidad de grasa a partir de los 7 años. 
En efecto, los resultados aluden que el sexo/género y la edad son variables que predicen la obesidad en hombres en edad escolar, leído de esta manera, se puede inferir que las mujeres pueden presentar una menor probabilidad de padecer enfermedades crónico-degenerativas. Empero, la prevalencia de sobrepeso y la obesidad fue significativamente mayor en hombres; no se observó diferencia significativa en las medias de IMC por grupos de edad y género, lo que inspira realizar estudios que requieren mayor profundidad y con perspectivas teóricas holísticas para evitar falsas conclusiones.

Sin duda, las prevalencias de sobrepeso y obesidad observadas en este estudio son similares a las de escolares de origen hispano en New York realizada en el 2004 por Thorpe et al., (2004) la cual reportó un 31\% de sobrepeso y obesidad, mientras que la Encuesta NHANES 2003-2004 reveló 16\% de sobrepeso y obesidad (Ogden et al., 2006).

Pero, también, es cierto que este estudio muestra sus propios limitantes explicativos, por ejemplo; la estatura baja en infantes se ha relacionado en otros estudios con el origen étnico (véase Castillo y Zenteno, 2004) y se tiene conocimiento que la estatura baja es el sacrificio que hacen los individuos para conservar el peso ante una dieta deficiente en proteínas y calorías (Halac y Zimmerman, 2004), y al no existir información del dato de origen étnico para la población estudiada, podríamos estar infiriendo argumentos no verídicos, por lo que se sugiere seguir realizando estudios antropométricos para contar con datos más confiables sobre las transiciones nutricionales de estatura baja y obesidad.

En lo que respecta a la media del IMC de la población estudiada, encontramos que fue de 21.39, lo que corresponde a la normalidad (índice deseado), pero, desafortunadamente, este resultado no hace evidente el porcentaje de grasa corporal que presenta el individuo, convirtiéndolo en un método subjetivo de valoración por sí solo. Varios autores (Moreno et al., 2002; McCarthy, 2003, Ekelund et al., 2006), aseveran que el IMC solo puede subestimar la proporción de obesidad y que no es suficiente como indicador de riesgo de alteraciones metabólicas, porque no discrimina la masa grasa de la magra en un individuo, por lo cual la evaluación antropométrica debería complementarse con la circunferencia de cintura (CC) (Daniels, 2000). Si bien en este estudio no se tomaron medidas de $\mathrm{CC}$ en preescolares y escolares de primaria, sí se realizó a los escolares de la secundaria, donde se observó que el $\mathrm{CC}$ fue de $75.27 \mathrm{~cm}$ en promedio para escolares con origen Otomí, y de $78.73 \mathrm{~cm}$ para quienes no tienen antecedentes étnicos, estos datos no representan riesgos de complicaciones metabólicas, aunque están muy cerca de presentarlos considerando que el parámetro de alerta de $\mathrm{CC}$ en adolescentes es de $80 \mathrm{~cm}$. (Gower y Freedman, 1999; Moreno et al.. 2002)

Del mismo orden de preocupaciones, existe certeza de que en la niñez se encuentran presentes enfermedades cardiovasculares en relación con el tejido adiposo visceral (Owens, 1998), esto también se asocia con valores alterados de insulina y glucosa en sangre (intolerancia a la insulina, DM) (Gower y Freedman, 1999). Evidentemente, la medición de la circunferencia de cintura es una herramienta sencilla y útil, que permite optimizar los recursos económicos disponibles para detectar preescolares y escolares con mayor riesgo de padecer complicaciones metabólicas.

Un estudio realizado en escolares (McCarthy et al., 2003,) demostró que el incremento de sobrepeso y obesidad con base en grasa visceral implica un riesgo mayor de padecer enfermedades crónico-degenerativas, lo cual indica que realizar la medición de CC debe ser una 
medida obligatoria para incluirla en la rutina de la atención. Vague (1998) hace referencia que en 1956 se observó que existía un patrón entre obesidad y una predisposición a diabetes mellitus tipo 2, ateroesclerosis y gota. De la misma manera, Lebovitz (2003) refiere que en estudios longitudinales realizados entre 1967 y 1982 la presencia de obesidad abdominal en escolares, predecía la aparición de diabetes mellitus, infarto al miocardio, angina de pecho, accidente cerebro vascular, e incluso la muerte.

\section{CONCLUSIONES}

La presente investigación tiene el objetivo de generar conocimiento relevante y útil para la prevención y control de la obesidad en preescolares y escolares de comunidades de origen indígena con características periurbanas.

Con medidas sencillas y periódicas se pueden detectar los casos que presentan riesgos para desarrollar enfermedades metabólicas. El IMC resulta ser un indicador útil para ello, pero no suficiente, es necesario incluir la circunferencia de cintura en la vigilancia nutricional desde la edad preescolar para, tamizar a aquellos individuos con obesidad central como marcador de riesgo de enfermedad cardiovascular.

Se sugiere poner en práctica estrategias para evitar el sobrepeso y la obesidad en los preescolares y escolares a través del fomento de estilos de vida saludables, tomando en cuenta los modos de vida locales y sus dinámicas cambiantes. La actividad física continua, buenos hábitos dietéticos, conciencia de la salud-enfermedad, conocimiento de lo que es el sobrepeso y la obesidad, entre otras prácticas contempladas en "paquetes" de salud integral, si bien son factores determinantes e intervinientes para lograr el máximo desarrollo potencial infantil (Solano et al., 2005), estos pueden verse limitados ante el desconocimiento de los usos, costumbres e hibridaciones de las culturas locales, sobretodo, de aquellas que están en un continuo proceso de transformación por su interacción con el crecimiento urbano y los consumos globales. 


\section{REFERENCIAS BIBLIOGRÁFICAS}

Angarita C., Machado D., Morales G., García G., Arteaga F., Silva T. y Alarcón O. (2001). Estado nutricional, antropométrico, bioquímico y clínico en preescolares de la comunidad rural de Canaguá. Estado Mérida. Anales Venezolanos de Nutrición, 14(2), 75-85.

Aseguinolaza, I., Callén, M., Esperanza, J., Ozcoidi, I. y Alustiza, E. (2001). Prevención y tratamiento de la obesidad infantil en atención primaria. Revista Española de Nutrición Comunitaria, 1(D), 192-196.

Bastos A., González R., Molinero O. y Salguero, A. (2005). Obesidad, nutrición y actividad física. Revista Internacional de Medicina y Ciencias de la Actividad Física y del Deporte. 18,18 .

Bernard L., Lavallée C., Gray-Donald K. y Delisle H. (1995). Overweight in Cree schoolchildren and adolescents associated with diet, low physical activity, and high television viewing. Journal of the American Diet Association, 95, 800-802.

Bustos P., Muñoz S., Vargas C. y H. Amigo (2009). Pobreza y procedencia indígena como factores de riesgo de problemas nutricionales de los niños que ingresan a la escuela. Salud Pública de México, 51(3),187-193.

Caballero E., Himes J., Lohman T., Davis S., Stevens J. y M. Evans (2003). Body composition and overweight prevalence in 1704 schoolchildren from 7 American Indian communities. American Journal of Clinic Nutrition, 78, 308-312.

Carmona M. e I. Vizcarra (2009). Obesidad en escolares de comunidades rurales con alta migración internacional en el México central. Población y salud en Mesoamérica, 6 (002), 2.

Castillo, J. L. y R. Zenteno (2004). Valoración del Estado Nutricional. Instituto de Salud Pública Universidad Veracruzana. Facultad de Nutrición Universidad Veracruzana. Vol 4. Núm. 2 Julio - Diciembre. s/p.

Cole T. (2004). Children grow and horses race: is the adiposity rebound a critical period for later obesity? BMC Pediatrics, 4, 6.

CONAPO (Consejo Nacional de Población) (2006). II Conteo de Población y Vivienda 2005, Principales resultados por localidad (ITER), INEGI, México.

CONAPO -INEGI (Instituto Nacional de Estadística, Geografía e Informática) (2011). Censo de Población y Vivienda 2010. Principales resultados por localidad (ITER), INEGI, México.

CONEVAL (Consejo Nacional de Evaluación de las Políticas de Desarrollo Social) (2009). Informe de evolución histórica de la situación nutricional de la población y los programas de alimentación, nutrición y abasto en México, SEDESOL. 
Conzuelo V. e I. Vizcarra (2009). Variables socionutricionales de hogares mazahuas integrados por preescolares desnutridos con madres con obesidad y sin obesidad. Población y Salud en Mesoamérica, 6(002).

Daniels, S. (2000). Utility of Different Measures of Body Fat Distribution in Children and Adolescents. Am J of Epidemiol; 152 (12):1179-84.

Dietz, W. (1997). Periods of risk in childhood for the development of adult obesity: what do we need to learn? The Journal of Nutrition, 27, 1884S-1886S.

Ekelund, U., Ong, K., Linne, Y., Neovius, M., Brage, S. y D.B. Dunger (2006). Upward weight percentile crossing in infancy and early childhood independently predicts fat mass in young adults: the Stockholm Weight Development Study (SWEDES). Am J Clin Nutr; 83 (2): 324-30.

Gotthelf S., Aramayo M. y S. Alderete (2004). Coexistencia de obesidad y desnutrición en familias pobres. Disponible en: http:// www. nutrinfo. com/ pagina /info/ ob04- 04.pdf.

Gower, D. y D. Freedman (1999). Relation of circumferences and skinfold thicknesses to lipid and insulin concentrations in children and adolescents: the Bogalusa Heart Study. Am J Clin Nutr; 69:308-17.

Halac, I. y D., Zimmerman (2004). Evaluating short stature in children. Pediatr Ann 2004; 33:170-176.

Halper, P. (2007). "Obesity and American Indians/Alaska Natives”. Reporte postdoctoral para U.S. Department of Health and Human Services Office of the Assistant Secretary for Planning and Evaluation.

Krebs N., John H., Dawn J., Theresa A., Guilday P. y D. Styne (2007). Assessment of Child and Adolescent Overweight and Obesity. Pediatrics, 120, S193-S228.

Lamas, Marta (2002), Cuerpo, diferencia sexual y género. Taurus, México.

Lebovitz, H.E. (2003). Resistencia a la insulina. SaoPaulo: Sciencie Press Ltda.

Malina R., Bouchard C. y O. Bar-Or (2004). Growth, Maturation and, Physical Activity. Human Kinetics Books, Champaing.

McCarthy, H.D., Ellis, S.M. y T.J. Cole (2003). Central overweight and obesity in British youth aged 11-16 years: cross sectional surveys of waist circumference. BMJ; 326 (7390): 624.

Mei Z., Grummer-Strawn L., Pietrobelli A., Goulding A., Goran M. y W. Dietz (2002). Validity of body mass index compared with other body-composition screening indexes for the assess-ment of body fatness in children and adolescents. American Journal of Clinic Nutrition, 75, $978-985$. 
Moreno, L.A., Pineda, I., Rodríguez, G., Fleta, J., Sarria, A. y M. Bueno (2002). Waist circunference for the screening of the metabolic syndrome in children. Acta Paediatr; 91 (12): 1307-12.

Nestle, M. (2007). Dietética elemental. Investigación y ciencia, 374, 20-29.

NIH (National Institutes of Health) (2009). Clinical Guidelines on the Identification, Evaluation, and Treatment of Overweight and Obesity in Adults. The Evidence Report. National Institutes of Health. Disponible línea: http://www.nhlbi.nih.gov/guidelines/obesity/ob_home.htm. Consultado en diciembre de 2010.

Ogden, C.L., Carrol, M.D., Curting, L.R., McDowell, M.A., Tabak, C.J. y K.M. Flegal(2006). Prevalence of overweight and obesity in the United States, 1999-2004. JAMA; 295: 154955.

Olaiz-Fernández, G. R., Shama-Levy, T, Rojas, R., Villalpando-Hernández y S. Ramírez-Silva (2006). Encuesta Nacional de Salud y Nutrición 2006. Cuernavaca Morelos: Instituto Nacional de Salud Pública.

Ortiz-Hernández, L. y L.I. Cruz-Ángeles (2005). Asociación del crecimiento físico con la composición corporal en escolares de Xochimilco. Bol. Med. Hosp. Infant. Mex. [revista en la Internet]. Consultado el 15 de Dic 2011; 62(6): 428-442. Disponible en: http://www.scielo.org.mx/scielo.php?

script $=$ sci_arttext\&pid=S16651146200500060000\&lng $=$ es

Owens, S. (1998). Visceral adipose tissue and cardiovascular risk factors in obese children. J Pediatr; 133 (1): 41-5

Padrón, M. (2002). Obesidad infantil: un problema creciente. Nutrición clínica. 5(4): 258-262.

Peña, M. y J. Bacallao (2000). La obesidad y la pobreza: un problema emergente en las Américas. En: La obesidad en la pobreza. Un nuevo reto para la salud pública. Organización Panamericana de la Salud. Oficina Sanitaria Panamericana. Organización Mundial de la Salud, 576, 3-11.

Pietrobelli A., Faith M., Allison D., Gallagher D., Chiumello G. y S. Heymsfield (1998). Body mass index as a measure of adiposity among children and adolescents: a validation study. The Journal of Pediatrics, 132, $204-210$.

Psacharopoulos G. y H. Patrinos (1994). Indigenous people and poverty in Latin America: an empirical analysis. World Bank Regional and Sectoral Studies. Disponible en: http://www.cosmovisionandina.org/archivos/ yachaywasi/ IndigenousPovertyLA.pdf.

Rolland-Cachera M., Deheeger M., Bellisle F., Sempe M., Guilloud-Bataille M. y E. Patois (1984). Adiposity rebound in children: a simple indicator for predicting obesity. American Journal of Clinical Nutrition, 39, $129-135$. 
SEDESOL (Secretaría de Desarrollo Social) (2012). Catálogo de localidades 2012. INEGI, México. Disponible en línea http://cat.microrregiones.gob.mx/catloc/contenido.aspx? clave $=151060062 \&$ tbl $=$ tb101. Consultado el 5 de marzo de 2012

Solano L., Barón M., y S. Del Real (2005). Situación nutricional de preescolares, escolares, y adolescentes de Valencia, Carabobo, Venezuela. Anales Venezolanos de Nutrición, 18 (1), $72-76$.

Thorpe, L.E., List, D.G., Marx, T., May, L., Helgerson, S.D. y T.R. Frieden (2004). Childhood obesity in New York City elementary school students. Am J Public Health; 94: 1496-500.

Vague J (1998). Obesities. London: John Libbey Company Ltda.

Violante, R. (2001). Obesidad y diabetes tipo 2 en el niño. Una nueva epidemia Revista de Endocrinología y Nutrición, 9 (2), 103-106.

Vizcarra, I. y B. Lutz (2010). Globalisation et crises alimentaires: remesas, sécurité alimentaire et pauvreté dan l'État de Mexico (centre du Mexique), en Labrecque, M.F., M. Boulianne y S. Doyon Migration, environnement, violence et mouvements sociaux au Mexique. Dynamiques regionales en contexte d'économie globalisée. Press de la Universidad Laval, red Dialog, Quebec, 57-97. 


\section{AGRADECIMIENTOS}

Se agradece particularmente la colaboración incondicional al personal directivo y a las y los profesores de las escuelas: Jardín de Niños "Lic. Benito Juárez"; Escuela Primaria "Miguel Hidalgo y Costilla y de la Escuela Secundaria Técnica Industrial y Comercial No. 69 "Gral. Vicente Guerrero", que permitieron con interés la realización de este estudio dentro de las instalaciones escolares. 
Cuadro 1. Población estudiada por escuela y sexo/género.

\begin{tabular}{|c|c|c|c|c|}
\hline Escuela & $\begin{array}{c}\text { Total de } \\
\text { estudiantes }\end{array}$ & $\begin{array}{c}\text { Estudiantes } \\
\text { medidos }\end{array}$ & Porcentaje & $\begin{array}{c}\text { Porcentaje por } \\
\text { sexo/género de } \\
\text { estudiantes } \\
\text { medidos }\end{array}$ \\
\hline $\begin{array}{c}\text { Jardín de Niños } \\
\text { "Lic. Benito Juárez" }\end{array}$ & 349 & 305 & $87.39 \%$ & $\begin{array}{c}49.28 \% \text { mujeres } \\
50.72 \% \text { hombres }\end{array}$ \\
\hline $\begin{array}{c}\text { Escuela Primaria } \\
\text { "Miguel Hidalgo y Costilla" }\end{array}$ & 1268 & 1247 & $98.34 \%$ & $\begin{array}{c}50.39 \% \text { mujeres } \\
49.61 \% \text { hombres }\end{array}$ \\
\hline $\begin{array}{c}\text { Escuela Secundaria Técnica } \\
\text { Industrial y Comercial No. } \\
69 \text { "Gral. Vicente Guerrero" } \\
\text { (ESTIC) }\end{array}$ & 714 & 656 & $91.87 \%$ & $\begin{array}{c}52.13 \% \text { mujeres } \\
47.87 \% \text { hombres }\end{array}$ \\
\hline
\end{tabular}

Fuente: A partir de nuestros datos recolectados en trabajo de campo, 2010. 


\section{Cuadro 2. Datos antropométricos y estadísticos de la población estudiada por escuela y} sexo/género

\begin{tabular}{|c|c|c|c|c|c|}
\hline Escuela & $\begin{array}{l}\text { Sexo/ } \\
\text { género }\end{array}$ & Variable & $\mathbf{N}$ & Media & Desviación Estándar \\
\hline \multirow{8}{*}{$\begin{array}{l}\text { Jardín de Niños } \\
\text { "Lic. Benito Juárez" }\end{array}$} & \multirow{4}{*}{$\mathrm{F}$} & Edad & 171 & 5.06 & 0.73 \\
\hline & & IMC & 154 & 15.96 & 1.31 \\
\hline & & Peso & 154 & 18.37 & 2.65 \\
\hline & & Estatura & 154 & 1.07 & 0.06 \\
\hline & \multirow{4}{*}{ M } & Edad & 177 & 5.06 & 0.68 \\
\hline & & IMC & 151 & 16.18 & 1.63 \\
\hline & & Peso & 151 & 19.08 & 3.26 \\
\hline & & Estatura & 151 & 1.08 & 0.06 \\
\hline \multirow{8}{*}{$\begin{array}{c}\text { Escuela Primaria } \\
\text { "Miguel Hidalgo y } \\
\text { Costilla" }\end{array}$} & \multirow{4}{*}{$\mathrm{F}$} & Edad & 639 & 8.38 & 1.71 \\
\hline & & IMC & 628 & 17.61 & 2.73 \\
\hline & & Peso & 579 & 29.32 & 8.71 \\
\hline & & Estatura & 629 & 1.45 & 4.62 \\
\hline & \multirow{4}{*}{ M } & Edad & 629 & 8.61 & 1.68 \\
\hline & & IMC & 619 & 18.13 & 2.92 \\
\hline & & Peso & 578 & 30.82 & 9.08 \\
\hline & & Estatura & 619 & 1.28 & 0.12 \\
\hline \multirow{10}{*}{ ESTIC } & \multirow{5}{*}{$\mathrm{F}$} & Edad & 342 & 13.43 & 0.98 \\
\hline & & IMC & 342 & 22.14 & 3.29 \\
\hline & & Peso & 342 & 50.67 & 8.97 \\
\hline & & Estatura & 342 & 1.51 & 0.05 \\
\hline & & Cintura & 342 & 75.58 & 7.91 \\
\hline & \multirow{5}{*}{ M } & Edad & 314 & 13.57 & 1.03 \\
\hline & & IMC & 314 & 20.80 & 3.45 \\
\hline & & Peso & 314 & 51.48 & 10.89 \\
\hline & & Estatura & 314 & 1.57 & 0.08 \\
\hline & & Cintura & 314 & 75.24 & 24.25 \\
\hline
\end{tabular}

Fuente: Estimaciones en base a datos primarios. 
Cuadro 3. Diagnóstico nutricional y datos antropométricos de los preescolares del Jardín de Niños "Lic. Benito Juárez".

\begin{tabular}{|c|c|c|c|c|}
\hline \multicolumn{5}{|c|}{ Jardin de Ninos "Lic. Benito Juarez". } \\
\hline Diagnóstico & $\mathbf{N}$ & Variable & Media & Desviación Estándar \\
\hline \multirow{4}{*}{ p5 bajo peso } & \multirow{4}{*}{5} & Edad & 5.00 & 0.71 \\
\hline & & IMC & 13.42 & 0.55 \\
\hline & & Peso & 15.86 & 0.82 \\
\hline & & Estatura & 1.09 & 0.04 \\
\hline \multirow{4}{*}{ p50 normal } & \multirow{4}{*}{$\begin{array}{c}22 \\
5\end{array}$} & Edad & 5.07 & 0.72 \\
\hline & & IMC & 15.49 & 0.75 \\
\hline & & Peso & 17.92 & 2.21 \\
\hline & & Estatura & 1.07 & 0.06 \\
\hline \multirow{4}{*}{$\begin{array}{c}\mathrm{p} 85 \\
\text { sobrepeso }\end{array}$} & \multirow{4}{*}{54} & Edad & 4.94 & 0.66 \\
\hline & & IMC & 17.25 & 0.39 \\
\hline & & Peso & 20.04 & 2.54 \\
\hline & & Estatura & 1.08 & 0.06 \\
\hline \multirow{4}{*}{ p95 obesidad } & \multirow{4}{*}{21} & Edad & 5.14 & 0.73 \\
\hline & & IMC & 19.82 & 1.55 \\
\hline & & Peso & 24.64 & 3.46 \\
\hline & & Estatura & 1.11 & 0.06 \\
\hline
\end{tabular}

Fuente: Estimaciones en base a datos primarios.

Cuadro 4. Diagnóstico nutricional de los preescolares del Jardín de Niños

"Lic. Benito Juárez" por sexo/género.

\begin{tabular}{|c|c|c|c|c|c|}
\hline \multirow{2}{*}{ Diagnóstico } & \multicolumn{3}{|c|}{ Sexo/género } & \multirow{2}{*}{ Total } \\
\cline { 2 - 6 } & \multicolumn{2}{|c|}{ F } & \multicolumn{2}{c|}{ M } & \\
\cline { 2 - 6 } & Frecuencia & Porcentaje & Frecuencia & Porcentaje & \\
\hline p5 bajo peso & 1 & 0.65 & 4 & 2.65 & 5 \\
\hline p50 normal & 116 & 75.32 & 109 & 72.19 & 225 \\
\hline p85 sobrepeso & 31 & 20.13 & 23 & 15.23 & 54 \\
\hline p95 obesidad & 6 & 3.90 & 15 & 9.93 & 21 \\
\hline Total & 154 & 100 & 151 & 100 & 305 \\
\hline
\end{tabular}

Fuente: Estimaciones en base a datos primarios. 
Cuadro 5. Análisis de las estimaciones de máxima probabilidad (modelo de regresión logística) de los preescolares del Jardín de Niños "Lic. Benito Juárez".

\begin{tabular}{|c|c|c|c|c|}
\hline Variable & $\begin{array}{c}\text { Parámetro } \\
\text { Estimado }\end{array}$ & $\begin{array}{c}\text { Error } \\
\text { Estándar }\end{array}$ & $\begin{array}{c}\text { Wald } \\
\text { Chi-cuadrada }\end{array}$ & $\operatorname{Pr}>\mathbf{C h i}^{\mathbf{2}}$ \\
\hline Intercepto & -2.2046 & 0.2721 & 65.6621 & $<.0001$ \\
\hline Mujer & -1.0008 & 0.4974 & 4.0481 & $\underline{0.0442}$ \\
\hline
\end{tabular}

Fuente: Estimaciones en base a datos primarios.

Cuadro 6. Diagnóstico nutricional y datos antropométricos de los escolares de la Escuela Primaria "Miguel Hidalgo y Costilla"

\begin{tabular}{|c|c|c|c|c|}
\hline Diagnóstico & Variable & $\mathbf{N}$ & Media & $\begin{array}{c}\text { Desviación } \\
\text { Estándar }\end{array}$ \\
\hline \multirow{4}{*}{ p5 bajo peso } & Edad & 19 & 8.42 & 1.80 \\
\cline { 2 - 5 } & IMC & 19 & 13.21 & 0.99 \\
\cline { 2 - 5 } & Peso & 18 & 23.31 & 6.73 \\
\cline { 2 - 5 } & Estatura & 19 & 1.31 & 0.19 \\
\hline \multirow{4}{*}{ p50 normal } & Edad & 854 & 8.42 & 1.71 \\
\cline { 2 - 5 } & IMC & 854 & 16.61 & 1.42 \\
\cline { 2 - 5 } & Peso & 782 & 27.31 & 6.58 \\
\cline { 2 - 5 } & Estatura & 854 & 1.26 & 0.12 \\
\hline \multirow{4}{*}{ p85 sobrepeso obesidad } & Edad & 234 & 8.53 & 1.71 \\
\cline { 2 - 5 } & IMC & 234 & 19.59 & 1.62 \\
\cline { 2 - 5 } & Peso & 223 & 33.30 & 8.20 \\
\cline { 2 - 5 } & Estatura & 234 & 1.29 & 0.11 \\
\cline { 2 - 5 } & Edad & 140 & 8.94 & 1.55 \\
\cline { 2 - 5 } & IMC & 140 & 23.32 & 2.73 \\
\cline { 2 - 5 } & Peso & 133 & 41.83 & 10.64 \\
\hline & Estatura & 140 & 1.32 & 0.11 \\
\hline
\end{tabular}

Fuente: Estimaciones en base a datos primarios.

Cuadro 7. Diagnóstico nutricional de los escolares de la Escuela Primaria "Miguel Hidalgo y Costilla" por sexo/género.

\begin{tabular}{|c|c|c|c|c|c|}
\hline \multirow{2}{*}{ Diagnóstico } & \multicolumn{3}{|c|}{ Sexo/género } & \multirow{2}{*}{ Total } \\
\cline { 2 - 5 } & \multicolumn{2}{|c|}{ F } & \multicolumn{2}{c|}{ M } & \\
\cline { 2 - 5 } & Frecuencia & Porcentaje & Frecuencia & Porcentaje & \\
\hline p5 bajo peso & 12 & 1.91 & 7 & 1.13 & 19 \\
\hline p50 normal & 467 & 74.36 & 387 & 62.52 & 854 \\
\hline p85 sobrepeso & 100 & 15.92 & 134 & 21.65 & 234 \\
\hline p95 obesidad & 49 & 7.80 & 91 & 14.70 & 140 \\
\hline Total & 628 & 100 & 619 & 100 & 1247 \\
\hline
\end{tabular}

Fuente: Estimaciones en base a datos primarios. 
Cuadro 8. Análisis de las estimaciones de máxima probabilidad (modelo de regresión logística) de los escolares de la Escuela Primaria "Miguel Hidalgo y Costilla"

\begin{tabular}{|c|c|c|c|c|}
\hline Variable & $\begin{array}{c}\text { Parámetro } \\
\text { Estimado }\end{array}$ & $\begin{array}{c}\text { Error } \\
\text { Estándar }\end{array}$ & $\begin{array}{c}\text { Wald } \\
\text { Chi-cuadrada }\end{array}$ & Pr $>\mathbf{C h i}^{2}$ \\
\hline Intercepto & -1.7582 & 0.1135 & 239.9603 & $<.0001$ \\
\hline Mujer & -0.7112 & 0.1871 & 14.4459 & $\underline{0.0001}$ \\
\hline
\end{tabular}

Fuente: Estimaciones en base a datos primarios..

Cuadro 9. Diagnóstico nutricional y datos antropométricos de los escolares de la ESTIC

\begin{tabular}{|c|c|c|c|c|}
\hline Diagnóstico & Observaciones & Variable & Media & Desviación Estándar \\
\hline \multirow{5}{*}{ p5 bajo peso } & \multirow{5}{*}{ 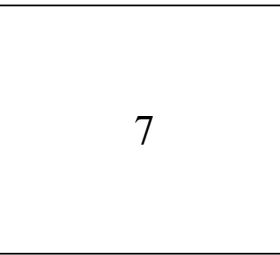 } & Edad & 13.71 & 1.38 \\
\hline & & IMC & 15.38 & 0.91 \\
\hline & & Peso & 38.13 & 6.33 \\
\hline & & Estatura & 1.57 & 0.09 \\
\hline & & Cintura & 64.36 & 3.38 \\
\hline \multirow{5}{*}{ p50 normal } & \multirow{5}{*}{430} & Edad & 13.52 & 0.96 \\
\hline & & IMC & 19.68 & 1.85 \\
\hline & & Peso & 46.78 & 6.68 \\
\hline & & Estatura & 1.54 & 0.08 \\
\hline & & Cintura & 71.61 & 20.10 \\
\hline \multirow{5}{*}{ p85 sobrepeso } & \multirow{5}{*}{149} & Edad & 13.42 & 1.08 \\
\hline & & $\mathrm{IMC}$ & 23.94 & 1.35 \\
\hline & & Peso & 56.07 & 6.21 \\
\hline & & Estatura & 1.53 & 0.06 \\
\hline & & Cintura & 80.06 & 5.06 \\
\hline \multirow{5}{*}{ p95 obesidad } & \multirow{5}{*}{70} & Edad & 13.51 & 1.06 \\
\hline & & IMC & 28.07 & 2.29 \\
\hline & & Peso & 67.95 & 9.92 \\
\hline & & Estatura & 1.55 & 0.08 \\
\hline & & Cintura & 90.06 & 6.48 \\
\hline
\end{tabular}

Fuente: Estimaciones en base a datos primarios.

Cuadro 10. Diagnóstico nutricional de los escolares de la ESTIC por sexo/género.

\begin{tabular}{|c|c|c|c|c|c|}
\hline \multirow{2}{*}{ Diagnóstico } & \multicolumn{4}{|c|}{ Sexo/género } & \multirow{2}{*}{ Total } \\
\cline { 2 - 5 } & \multicolumn{2}{|c|}{ F } & \multicolumn{2}{c|}{ M } & \\
\cline { 2 - 5 } & Frecuencia & Porcentaje & Frecuencia & Porcentaje & \\
\hline p5 bajo peso & 2 & 0.58 & 5 & 1.59 & 7 \\
\hline p50 normal & 206 & 60.23 & 224 & 71.34 & 430 \\
\hline p85 sobrepeso & 100 & 29.24 & 49 & 15.61 & 149 \\
\hline p95 obesidad & 34 & 9.94 & 36 & 11.46 & 70 \\
\hline Total & 342 & 100 & 314 & 100 & 656 \\
\hline
\end{tabular}

Fuente: Estimaciones en base a datos primarios. 
Cuadro 11. Análisis de las estimaciones de máxima probabilidad (modelo de regresión logística) de los escolares de la ESTIC por sexo/género y edad.

\begin{tabular}{|c|c|c|c|c|}
\hline Variable & $\begin{array}{c}\text { Parámetro } \\
\text { Estimado }\end{array}$ & $\begin{array}{c}\text { Error } \\
\text { Estándar }\end{array}$ & $\begin{array}{c}\text { Wald } \\
\text { Chi-cuadrada }\end{array}$ & Pr $>\mathbf{C h i}^{2}$ \\
\hline Intercepto & -2.2055 & 1.7192 & 1.6457 & 0.1995 \\
\hline Mujer & -0.1579 & 0.2537 & 0.3875 & $\underline{0.5336}$ \\
\hline Edad & 0.0119 & 0.1259 & 0.0089 & $\underline{0.9248}$ \\
\hline
\end{tabular}

Fuente: Estimaciones en base a datos primarios.

Cuadro 12. Presencia de origen Otomí y datos antropométricos de los escolares de la ESTIC

\begin{tabular}{|c|c|c|c|c|c|}
\hline Origen Otomí & Observaciones & Porcentaje & Variable & Media & Desviación Estándar \\
\hline \multirow{5}{*}{$-8-200$} & \multirow{5}{*}{29} & \multirow{5}{*}{$4.42 \%$} & Edad & 13.41 & 1.05 \\
\hline & & & IMC & 23.95 & 4.85 \\
\hline & & & Peso & 56.38 & 13.65 \\
\hline & & & Estatura & 1.53 & 0.07 \\
\hline & & & Cintura & 78.74 & 10.24 \\
\hline \multirow{5}{*}{$\mathrm{Si}$} & \multirow{5}{*}{627} & \multirow{5}{*}{$95.58 \%$} & Edad & 13.50 & 1.00 \\
\hline & & & IMC & 21.39 & 3.31 \\
\hline & & & Peso & 50.81 & 9.67 \\
\hline & & & Estatura & 1.54 & 0.07 \\
\hline & & & Cintura & 75.27 & 17.97 \\
\hline
\end{tabular}

Fuente: Estimaciones en base a datos primarios.

Cuadro 13. Estimación de Parámetros (modelo de regresión lineal)para IMC de los escolares de la ESTIC

\begin{tabular}{|c|c|c|c|c|}
\hline Variable & $\begin{array}{c}\text { Parámetro } \\
\text { Estimado }\end{array}$ & $\begin{array}{c}\text { Error } \\
\text { Estándar }\end{array}$ & Valor de t & $\begin{array}{c}\text { Valor de p } \\
\mathbf{P}>|\mathbf{t}|\end{array}$ \\
\hline Intercepto & 13.54688 & 1.81993 & 7.44 & $<.0001$ \\
\hline Otomí & -2.57963 & 0.61818 & -4.17 & $\leq .0001$ \\
\hline Edad & 0.71661 & 0.12681 & 5.65 & $\leq .0001$ \\
\hline Mujer & 1.42764 & 0.25498 & 5.60 & $\leq .0001$ \\
\hline
\end{tabular}

Fuente: Estimaciones en base a datos primarios.

Cuadro 14. Análisis de las estimaciones de máxima probabilidad (modelo de regresión logística) de los escolares con obesidad de la ESTIC

\begin{tabular}{|c|c|c|c|c|}
\hline Variable & $\begin{array}{c}\text { Parámetro } \\
\text { Estimado }\end{array}$ & $\begin{array}{c}\text { Error } \\
\text { Estándar }\end{array}$ & $\begin{array}{c}\text { Wald } \\
\text { Chi-cuadrada }\end{array}$ & $\mathbf{p}>\mathbf{C h i}^{\mathbf{2}}$ \\
\hline Intercepto & -0.9715 & 1.7656 & 0.3028 & 0.5821 \\
\hline Otomí & -1.4385 & 0.4241 & 11.5049 & $\underline{0.0007}$ \\
\hline Edad & 0.0199 & 0.1269 & 0.0246 & $\underline{0.8754}$ \\
\hline Mujer & -0.1724 & 0.2565 & 0.4516 & $\underline{0.5016}$ \\
\hline
\end{tabular}

Fuente: Estimaciones en base a datos primarios. 
Cuadro 15. IMC según edad y grado escolar.

\begin{tabular}{|c|c|c|c|c|c|c|c|}
\hline \multirow{2}{*}{ Escuela } & \multirow{2}{*}{ Grado } & \multirow{2}{*}{$\begin{array}{c}\text { Promedio } \\
\text { de edad } \\
\text { (años) }\end{array}$} & \multirow{2}{*}{$\begin{array}{l}\text { Promedio de } \\
\text { IMC }\end{array}$} & \multicolumn{2}{|c|}{$\begin{array}{c}\text { Diagnóstico } \\
\text { p85 sobrepeso }\end{array}$} & \multicolumn{2}{|c|}{$\begin{array}{l}\text { Diagnóstico } \\
\text { p95 obesidad }\end{array}$} \\
\hline & & & & Frecuencia & Porcentaje & Frecuencia & Porcentaje \\
\hline \multirow{2}{*}{$\begin{array}{l}\text { Jardín de } \\
\text { Niños } \\
\text { "Lic. } \\
\text { Benito } \\
\text { Juárez" }\end{array}$} & $2^{\circ}$ & 4.575 & 17.62573556 & $\begin{array}{c}32 \\
(19 \mathrm{~F}, 13 \mathrm{M})\end{array}$ & 21.62 & $\begin{array}{c}8 \\
(3 \mathrm{~F}, 5 \mathrm{M}) \\
\end{array}$ & 5.4 \\
\hline & $3^{\circ}$ & 5.48571 & 18.37033124 & $\begin{array}{c}22 \\
(12 \mathrm{~F}, 10 \mathrm{M})\end{array}$ & 14 & $\begin{array}{c}13 \\
(3 \mathrm{~F}, 10 \mathrm{M})\end{array}$ & 8.28 \\
\hline \multirow{6}{*}{$\begin{array}{c}\text { Escuela } \\
\text { Primaria } \\
\text { "Miguel } \\
\text { Hidalgo } \\
\text { y } \\
\text { Costilla" }\end{array}$} & $1^{0}$ & 6 & 17.9909493 & $\begin{array}{c}35 \\
(20 \mathrm{~F}, 15 \mathrm{M})\end{array}$ & 17.15 & $\begin{array}{c}8 \\
(4 \mathrm{~F}, 4 \mathrm{M})\end{array}$ & 3.92 \\
\hline & $2^{\circ}$ & 7.11268 & 19.2646675 & $\begin{array}{c}47 \\
(23 \mathrm{~F}, 24 \mathrm{M})\end{array}$ & 19.58 & $\begin{array}{c}24 \\
(11 \mathrm{~F}, 13 \mathrm{M})\end{array}$ & 10 \\
\hline & $3^{\circ}$ & 8.17568 & 20.5639586 & $\begin{array}{c}43 \\
(16 \mathrm{~F}, 27 \mathrm{M})\end{array}$ & 18.53 & $\begin{array}{c}31 \\
(12 \mathrm{~F}, 19 \mathrm{M})\end{array}$ & 13.36 \\
\hline & $4^{0}$ & 9.21667 & 21.3797732 & $\begin{array}{c}35 \\
(18 \mathrm{~F}, 17 \mathrm{M})\end{array}$ & 18.04 & $\begin{array}{c}25 \\
(4 \mathrm{~F}, 21 \mathrm{M}) \\
\end{array}$ & 12.88 \\
\hline & $5^{\circ}$ & 10 & 22.5697126 & $\begin{array}{c}36 \\
(10 \mathrm{~F}, 26 \mathrm{M})\end{array}$ & 18.36 & $\begin{array}{c}24 \\
(10 \mathrm{~F}, 14 \mathrm{M})\end{array}$ & 12.24 \\
\hline & $6^{0}$ & 11.0152 & 23.4879245 & $\begin{array}{c}38 \\
(13 \mathrm{~F}, 25 \mathrm{M})\end{array}$ & 20.87 & $\begin{array}{c}28 \\
(8 \mathrm{~F}, 20 \mathrm{M})\end{array}$ & 15.38 \\
\hline \multirow{3}{*}{ ESTIC } & $1^{o}$ & 12.4177 & 24.07312969 & $\begin{array}{c}59 \\
(41 \mathrm{~F}, 18 \mathrm{M}) \\
\end{array}$ & 26.22 & $\begin{array}{c}20 \\
(9 \mathrm{~F}, 11 \mathrm{M}) \\
\end{array}$ & 8.88 \\
\hline & $2^{\circ}$ & 13.4 & 25.5396964 & $\begin{array}{c}42 \\
(30 \mathrm{~F}, 12 \mathrm{M})\end{array}$ & 21 & $\begin{array}{c}23 \\
(12 \mathrm{~F}, 11 \mathrm{M}) \\
\end{array}$ & 11.5 \\
\hline & $3^{\circ}$ & 14.5733 & 26.27939066 & $\begin{array}{c}48 \\
(29 \mathrm{~F}, 19 \mathrm{M})\end{array}$ & 20.47 & $\begin{array}{c}27 \\
(13 \mathrm{~F}, 14 \mathrm{M})\end{array}$ & 11.68 \\
\hline
\end{tabular}

Fuente: Estimaciones en base a datos primarios.

\section{Gráfica 1. Promedios de IMC / Promedios de Edad}

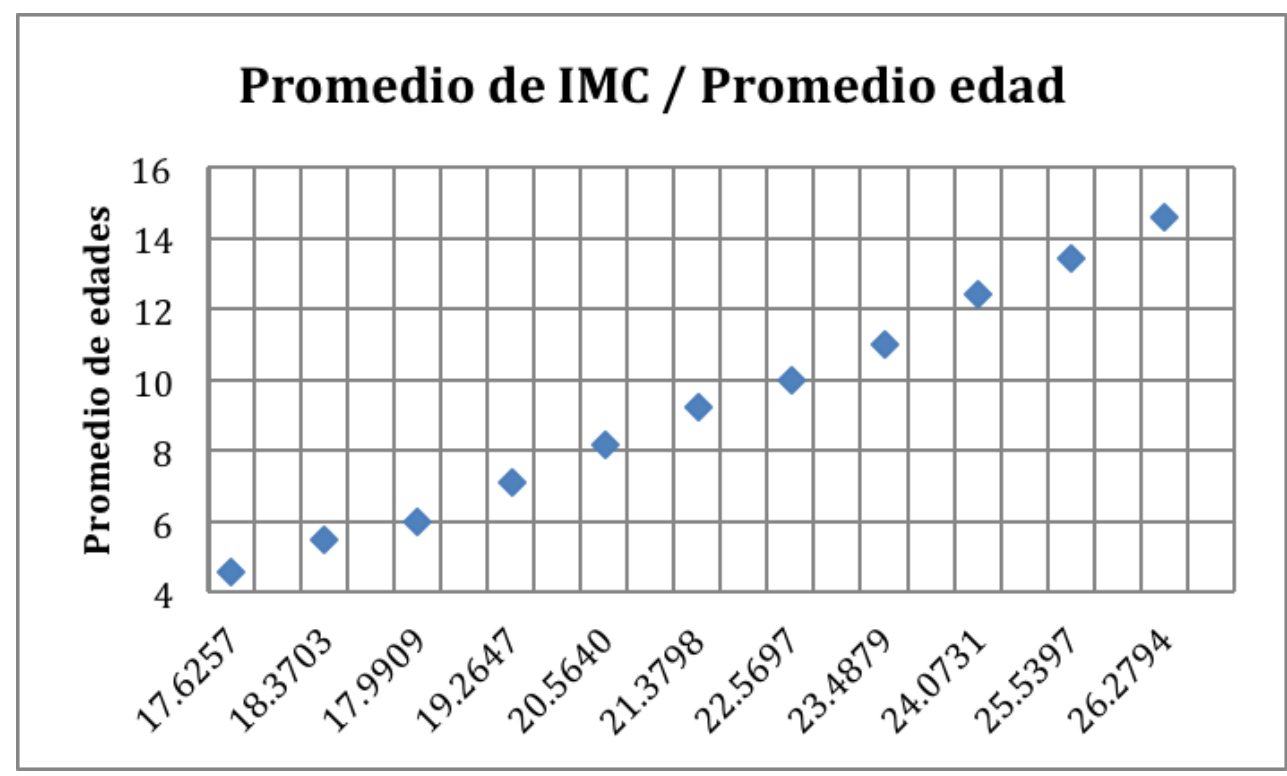

Fuente: Estimaciones en base a datos primarios. 